\title{
Motivations for fruit and vegetable consumption in the UK Women's Cohort Study
}

\author{
Jennie Pollard*, Darren Greenwood, Sara Kirk and Janet Cade \\ Nutrition Epidemiology Group, Nuffield Institute For Health, University of Leeds, 71-75 Clarendon Road, Leeds \\ LS2 9PL, UK
}

Received 18 July 2001; accepted 10 October 2001

\begin{abstract}
Background: Despite recommendations to increase fruit and vegetable consumption within the UK population, intakes are still too low. In order to stimulate dietary behaviour change the determinants of food choice need to be explored.

Objectives: To investigate how the priorities of high consumers of fruit and vegetables differ from those of low consumers, with respect to food choice motivations, and to identify what value high and low consumers place on different aspects of food choice behaviour.

Design: A 4-day food diary and a questionnaire, including The Food Choice Questionnaire (FCQ) and a measure of Stage of Change (SOC) for fruit and vegetable consumption, were administered to subjects. Motivation scores from the FCQ were compared with fruit and vegetable intake and SOC data.

Subjects: Nine hundred and ninety-eight females, aged 35-69 years, participating in the UK Women's Cohort Study.

Results: In a multiple linear regression model, including age, education level and all FCQ motivations, the strongest motivations specifically affecting fruit and vegetable intake were health and natural content. It was found that, for a one point increase (measured on a scale of 0-4) in health and natural content scores, fruit and vegetable consumption increased by 1.11 portions (95\% confidence interval (CI) $0.5-1.7$ ) and 0.84 portions $(95 \%$ CI $0.4-1.3)$, respectively $(P<0.01)$. The SOC evaluation showed significant associations with portions of fruit and vegetables consumed $(P<0.01)$. Women whose SOC was classed as maintenance phase were found to score higher on health, natural content, weight control and ethical concern factors $(P<0.01)$. These women also scored lower on convenience questions $(P<0.01)$.

Conclusion: In this particular group of women the most important motivating factors for food choice, within the high fruit and vegetable consumers, were health and natural content of the food.
\end{abstract}

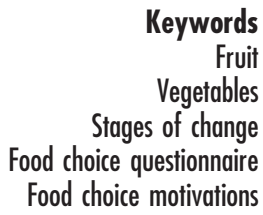

Fruit

egetables

ood choice questionnaire

Food choice motivations
In 1990 the World Health Organization recommended that a total intake of $400 \mathrm{~g}$ of fruit and vegetables should be included in the daily diet to provide protection against a number of diseases, such as cardiovascular disease and certain forms of cancer ${ }^{1}$. Intakes in the UK continue to fall short of this goal, particularly in certain subgroups of the population, despite health promotion attempts to increase consumption ${ }^{2,3}$. Adapting science and achieving meaningful behaviour change is a serious challenge for any health promotion programme. Social marketing is an approach that may be applied to the promotion of healthy eating, whereby programmes are directed at target groups in the population based on their differing attitudes, beliefs, motivations and behaviour ${ }^{4}$. Thus, in order to change the dietary behaviour of any population, it is important to take into consideration the motivations for food choice within that population.
The concept of applying social marketing techniques within dietary health promotion is not a new one; however, previous studies have concentrated their efforts on identifying barriers to change in low consumers of fruit and vegetables 5 . As more research is required to investigate how best to motivate population change, it is suggested that it would be useful to now identify factors that motivate current high consumers of fruit and vegetables.

The aim of the present study was to develop a greater understanding of the factors affecting food choice decisions in relation to fruit and vegetable consumption. The first objective was to investigate how the priorities of high consumers differ from those of low consumers with respect to food choice motivations and to identify what value high and low consumers place on different aspects of food choice behaviour. In order to do this The Food 
Choice Questionnaire (FCQ) was employed. This questionnaire was designed to measure the importance placed, by subjects, on nine different categories of food choice motivations: health, mood, convenience, sensory appeal, natural content, price, weight control, familiarity and ethical concern ${ }^{7}$.

The second objective of the study was to investigate the relationships between food choice motivations and the process of behaviour change. One model that enables us to better understand this process of behaviour change is the Transtheoretical Model (TTM). According to this model health-related behaviour change occurs through five separate stages, entitled The Stages of Change ${ }^{8}$. A measure of Stage of Change (SOC) for increasing fruit and vegetable consumption was therefore incorporated into the methodology.

\section{Subjects and methods}

\section{Study population}

The population for study was drawn from the UK Women's Cohort Study (UKWCS). The UKWCS is a 10 -year prospective study investigating cancer morbidity and mortality in women with a variety of dietary habits such as vegetarians, fish eaters and meat eaters. The cohort consists of 35000 women, across England, Wales, Scotland and Northern Ireland, aged between 35 and 69 years. The number of women selected from the cohort to receive an FCQ, in order to investigate food choice motivations for fruit and vegetable consumption, was decided using a power calculation based on previous studies that had utilised the $\mathrm{FCQ}^{7,9}$. Assuming a standard deviation of 0.8 , the number of subjects required to detect a difference of 0.3 , with $90 \%$ power, on any of the scales for the nine dimensions within the FCQ was 650. A significance level of 0.01 was used to allow for multiple comparisons. Allowing for a conservatively estimated response rate of 30\%, 2200 questionnaires were required to be sent. The UKWCS participants are contacted in waves and therefore the current study was based on one wave $(n=3001)$ of the UKWCS mailing that was sent out in August 1999.

\section{Data collection}

The 3001 women contacted each received a 4-day food diary and a questionnaire. The questionnaire included The Food Choice Questionnaire, which has been found to be reliable, internally consistent and stable over time $e^{7,9}$. This questionnaire begins with the sentence 'It is important to me that the food I eat on a typical day...' and is then followed by a series of 36 statements covering the nine dimensions measured by the FCQ. For example, 'It is important to me that the food I eat on a typical day is easy to prepare'. This statement is part of the assessment for the dimension of convenience. The participants are asked to tick an appropriate response for each statement ranging from 'not at all important' to 'very important' on a 4-point scale.

The second part of the questionnaire included an assessment of the Stage of Change (SOC) odel $^{8}$. Subjects were asked to read seven statements and to tick the box next to the statement that most applied to their diet. The statements related to whether or not the subjects were eating five portions of fruit and vegetables daily and, if so, how long they had been doing this for. Based on the response, subjects were classified into one of the five stages: pre-contemplation, contemplation, preparation, action or maintenance. Subjects classed in the Precontemplation phase were currently not consuming five portions of fruit and vegetables daily and did not want to start in the next six months. In the Contemplation phase subjects were still not consuming five portions but they stated that they did want to start in the next six months. In the next phase, Preparation, subjects were again not consuming five portions of fruit and vegetables daily but this time they stated that they would like to start in the next month. In the Action phase subjects were currently consuming five, or more, portions of fruit and vegetables daily, or on most days, and had been for less than six months. Finally, subjects in Maintenance were still currently consuming five, or more, portions daily, or on most days, but they had been doing this for more than six months.

\section{Statistical metbods and design}

Using the 4-day food diary data the numbers of portions of fruit and vegetables consumed per person were calculated and then averaged over the four-day period. This daily figure included fruit and vegetables from composite dishes and allowed for one portion of fruit juice per day but excluded potatoes. Standard portion sizes were employed for calculation of the amounts consumed ${ }^{10}$.

All analyses were carried out using the Statistical Package for the Social Sciences (SPSS) version 9.0 ${ }^{11}$. FCQ data were scored for each statement on the questionnaire so that scores ranged from 1 for an answer of 'not at all important' up to 4 for an answer of 'very important'. Scores on items contributing to each dimension were then added up and divided by the number of questions relating to that particular dimension, giving an average score on a scale of 1 to 4 , per person, for each of the nine dimensions ${ }^{7,9}$. Nonparametric tests were employed where necessary because of the skewed nature of the FCQ data. Average scores on the FCQ are presented as medians and correlations between FCQ factors were investigated using Spearman's correlation. The subjects were then split into tertiles of fruit and vegetable intake and median FCQ scores within each tertile were explored and compared using the KruskalWallis test. A linear regression model was then used to determine which of the FCQ factors were the strongest predictors of fruit and vegetable consumption. This was repeated adjusting for age and education level. 
Table 1 Sociodemographic and health behaviour characteristics of responders vs. non-responders

\begin{tabular}{lccr}
\hline & $\begin{array}{c}\text { Responders } \\
(n=998)\end{array}$ & $\begin{array}{c}\text { Non-responders } \\
(n=2003)\end{array}$ & $P$-value \\
\hline Median age (years) & 50.7 & 49.8 & $<0.05$ \\
Median BMI (kg m ${ }^{-2}$ ) & 23.8 & 23.9 & 0.32 \\
Median portions of fruit and vegetables consumed at baseline & 10.6 & 10.3 & 0.19 \\
Smokers & & 11.5 & $<0.01$ \\
Yes (\%) & 7.1 & & $<0.01$ \\
Vegetarian status & 47.5 & 41.0 & $<0.05$ \\
Vegetarian (\%) & & & \\
Marital status & 72.9 & 68.3 & \\
Married or living as married (\%) & 9.0 & 12.2 & \\
Divorced (\%) & 7.2 & 7.9 & \\
Widowed (\%) & 9.1 & 8.8 & \\
Single (\%) & 1.8 & 2.8 & \\
Separated (\%) & & & \\
Highest educational qualification & 13.0 & 18.0 & \\
No education (\%) & 28.9 & 30.5 & \\
'O'-level (\%) & 24.5 & \\
'A'-level (\%) & 31.7 & 27.0 & \\
Degree (\%) & & & \\
\hline
\end{tabular}

The SOC model was investigated to see if there were any relationships with median intakes of fruit and vegetables or median FCQ scores.

\section{Results}

\section{Descriptive statistics}

Out of the 3001 women contacted, 197 (6.6\%) had either died or had moved house. Some 1139 women completed and returned the FCQ and 1103 completed the 4-day food diary. Of these women 998 completed and returned both the diary and the questionnaire. Only women who had returned both were included in the analysis, giving an overall response rate of $36 \%$. Since baseline data were available on all subjects contacted the sociodemographic profiles of the responders and the non-responders could be compared and these are presented in Table 1. Responders were slightly older, less likely to be smokers and more likely to be vegetarian, married or living as married, with a degree level of education, compared with the non-responders. There was no significant difference between median portions of fruit and vegetables consumed per day, as assessed in the baseline analysis, using a food-frequency questionnaire.
The SOC evaluation showed significant associations with portions of fruit and vegetables consumed (Fig. 1), so that the number of portions of fruit and vegetables consumed daily increased as SOC moves towards the action and maintenance phases $(P<0.01)$.

The median scores for each of the FCQ factors are presented in Table 2, coupled with the results from the SOC analysis. It was found that within these women health, sensory appeal, natural content and weight control were the strongest determinants of general food choice in all subjects. Clear relationships can be also be observed between SOC and median FCQ scores. Women classed as being in the maintenance phase - that is, women who claim to have been eating five portions of fruit and vegetables on most days for more than six months - were found to score higher on the health, natural content, weight control and ethical concern factors than women in the other stages $(P<0.01)$. Conversely, women classed as being in the pre-contemplative phase - that is, women who stated that they were not consuming five portions of fruit and vegetables per day and did not intend to start in the next six months - had higher median scores for convenience factors than women in the other stages $(P<0.01)$.

Table 2 Median scores for each FCQ factor and SOC relationships with median FCQ scores

\begin{tabular}{|c|c|c|c|c|c|c|c|}
\hline & $\begin{array}{l}\text { All subjects } \\
(n=998)\end{array}$ & $\begin{array}{l}\text { Pre-contemplation } \\
\qquad(n=43)\end{array}$ & $\begin{array}{l}\text { Contemplation } \\
\quad(n=27)\end{array}$ & $\begin{array}{l}\text { Preparation } \\
(n=42)\end{array}$ & $\begin{array}{l}\text { Action } \\
(n=18)\end{array}$ & $\begin{array}{l}\text { Maintenance } \\
\quad(n=853)\end{array}$ & $P$-value \\
\hline Health & 3.33 & 3.00 & 3.00 & 3.00 & 3.00 & 3.33 & $<0.01$ \\
\hline Mood & 2.17 & 2.00 & 2.17 & 2.17 & 2.00 & 2.17 & 0.03 \\
\hline Convenience & 2.80 & 3.20 & 3.00 & 2.80 & 2.80 & 2.60 & $<0.01$ \\
\hline Sensory appeal & 3.25 & 3.38 & 2.88 & 3.25 & 3.25 & 3.25 & 0.46 \\
\hline Natural content & 3.33 & 3.00 & 2.67 & 3.00 & 3.00 & 3.33 & $<0.01$ \\
\hline Price & 2.67 & 2.67 & 2.67 & 2.67 & 3.00 & 2.67 & 0.09 \\
\hline Weight control & 3.00 & 2.67 & 2.67 & 2.67 & 2.67 & 3.00 & $<0.01$ \\
\hline Familiarity & 1.67 & 2.00 & 1.67 & 1.67 & 1.33 & 1.67 & 0.08 \\
\hline Ethical concern & 2.67 & 2.00 & 2.33 & 2.33 & 2.50 & 2.67 & $<0.01$ \\
\hline
\end{tabular}




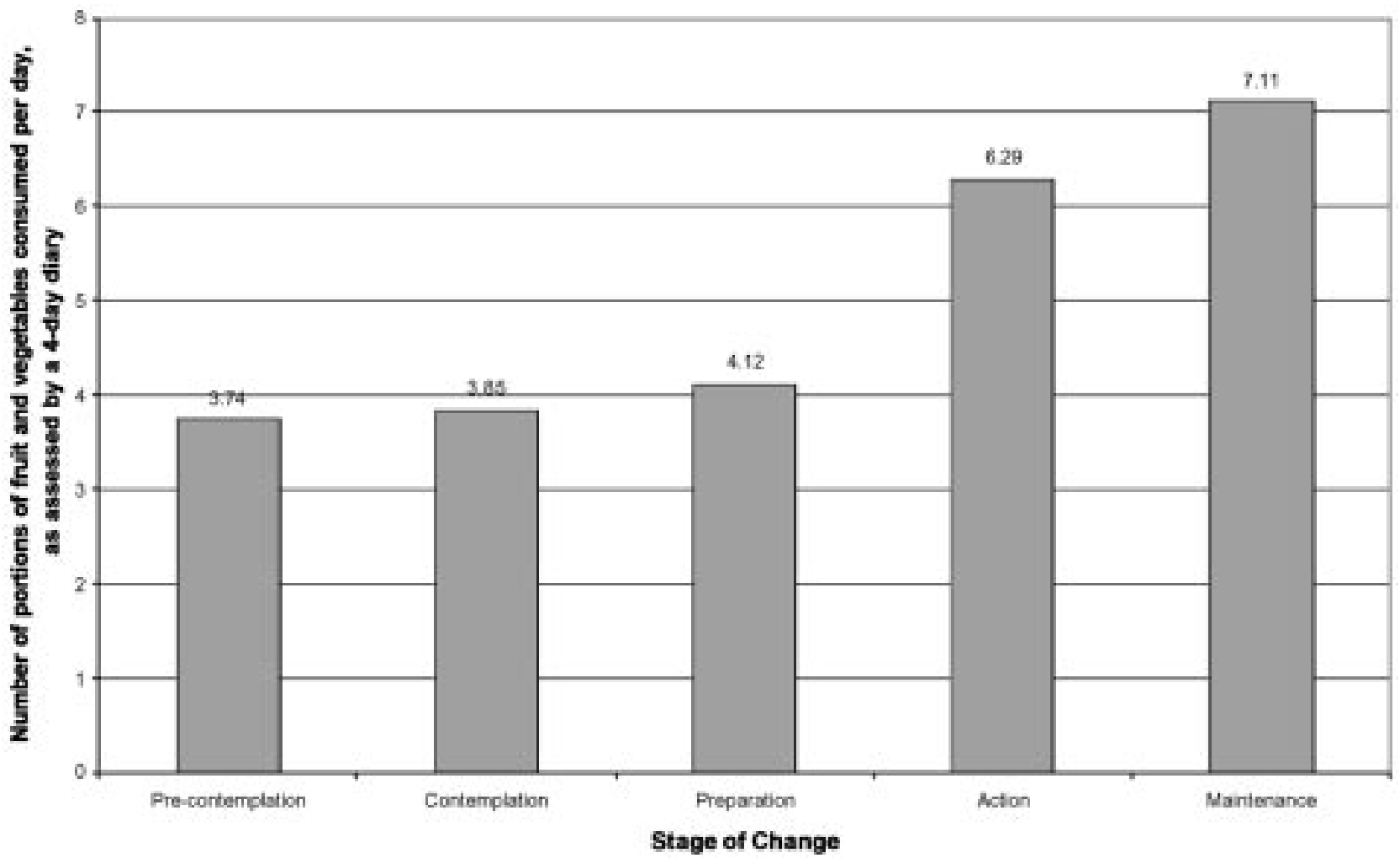

Fig. 1 Median portions of fruit and vegetables for each stage of change

\section{Univariate analysis}

Median scores on each FCQ scale according to tertile of fruit and vegetable intake can be seen in Fig. 2. Those in the highest tertile of fruit and vegetable intakes had higher median health, natural content and ethical concern scores compared with women in the lowest tertile of intakes $(P<$ 0.01). However, women appearing in the lowest tertile of intakes had a higher median score for the familiarity factor compared with the women in the highest tertile $(P<0.01)$.

\section{Multivariate analysis}

A linear regression model was employed to describe the impact of the nine FCQ factors on fruit and vegetable consumption. The unadjusted results may be seen in Table 3. The model was then repeated adjusting for age,

Table 3 Unadjusted results from the linear regression model describing the impact of the nine FCQ factors on fruit and vegetable consumption

\begin{tabular}{lcrrr}
\hline & $n$ & $\begin{array}{c}\text { Regression } \\
\text { slope }\end{array}$ & \multicolumn{1}{c}{$95 \% \mathrm{Cl}$} & $P$-value \\
\hline Health & 968 & 1.29 & 0.85 to 1.72 & $<0.01$ \\
Mood & 963 & 0.00 & -0.23 to 0.36 & 0.67 \\
Convenience & 961 & -0.26 & -0.57 to 0.05 & 0.10 \\
Sensory appeal & 969 & -0.14 & -0.53 to 0.25 & 0.48 \\
Natural content & 965 & 1.17 & 0.87 to 1.48 & $<0.01$ \\
Price & 972 & -0.27 & -0.57 to 0.02 & 0.07 \\
Weight control & 967 & 0.23 & -0.05 to 0.50 & 0.11 \\
Familiarity & 968 & -0.56 & -0.89 to -0.22 & $<0.01$ \\
Ethical concern & 987 & 0.36 & 0.11 to 0.62 & $<0.01$ \\
\hline
\end{tabular}

education level and all of the FCQ factors, and these results can be seen in Table 4 . The results show that the strongest motivations affecting specifically fruit and vegetable consumption were health and natural content. It was found that for a one point increase (measured on a scale of 0-4) in health and natural content scores, combined fruit and vegetable consumption increased by 1.11 portions (95\% confidence interval (CI) $0.5-1.7$ ) and 0.84 portions (95\% CI $0.4-1.3$ ), respectively.

\section{Discussion}

Respondents to the study seemed to be a slightly more 'health-conscious' group than the non-responders, being

Table 4 Results from the linear regression model describing the impact of the nine FCQ factors on fruit and vegetable consumption adjusted for age, education level and all of the FCQ factors $(n=867)$

\begin{tabular}{lrrr}
\hline & $\begin{array}{c}\text { Regression } \\
\text { slope }\end{array}$ & \multicolumn{1}{c}{$95 \% \mathrm{Cl}$} & $P$-value \\
\hline Health & 1.11 & 0.53 to 1.70 & $<0.01$ \\
Mood & -0.10 & -0.47 to 0.26 & 0.58 \\
Convenience & 0.01 & -0.42 to 0.31 & 0.78 \\
Sensory appeal & -0.40 & -0.82 to 0.03 & 0.07 \\
Natural content & 0.84 & 0.44 to 1.25 & $<0.01$ \\
Price & -0.17 & -0.51 to 0.17 & 0.32 \\
Weight control & 0.15 & -0.16 to 0.46 & 0.33 \\
Familiarity & -0.25 & -0.63 to 0.14 & 0.22 \\
Ethical concern & -0.01 & -0.40 to 0.23 & 0.60 \\
\hline
\end{tabular}




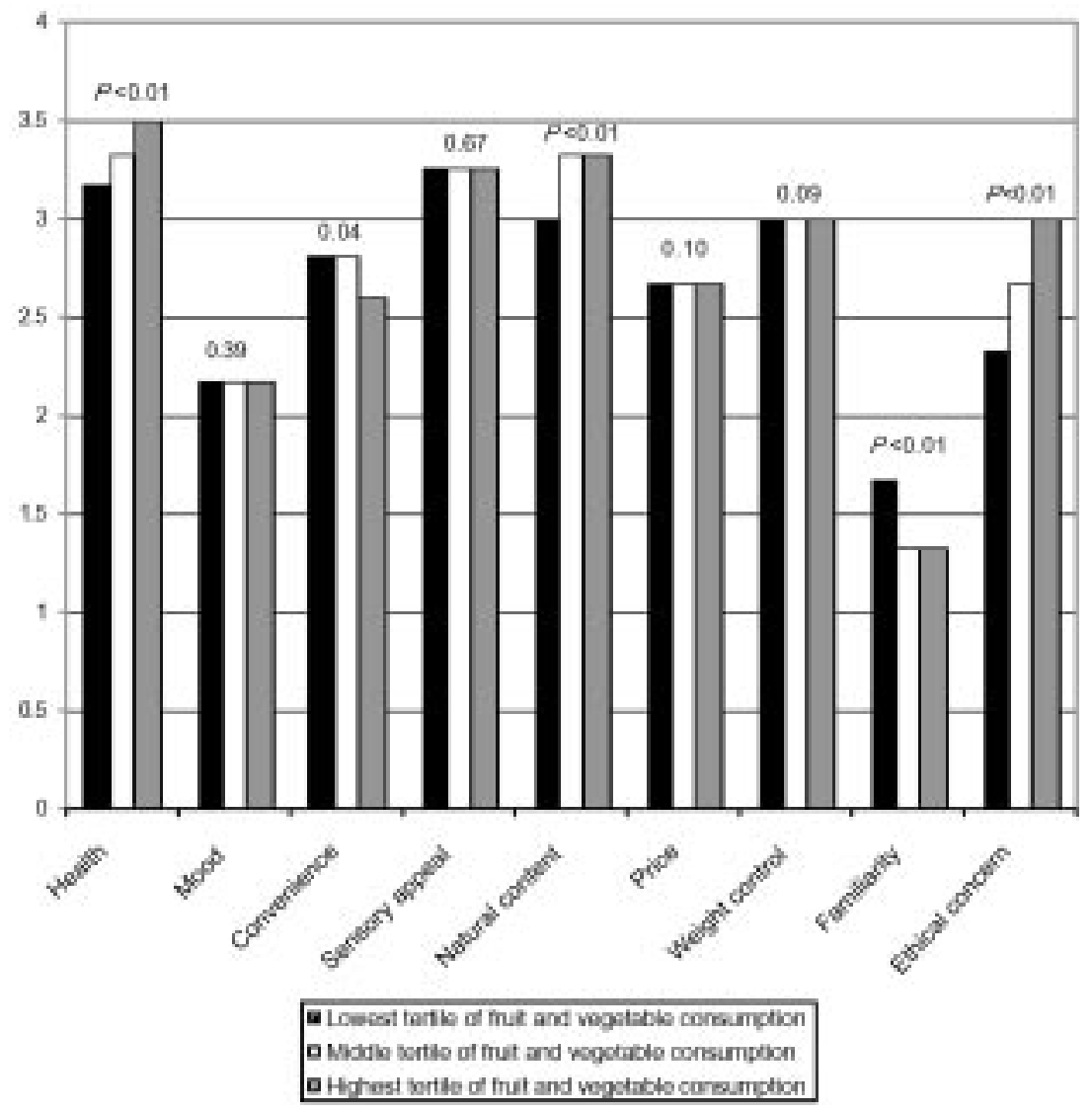

Fig. 2 Median scores on each FCQ scale according to tertile of fruit and vegetable intake

more likely to be non-smokers and vegetarians. However, when the median portions of fruit and vegetables consumed per day, at baseline, were compared, no significant differences were observed. Taking into account that the baseline data were collected between one and four years prior to this study, even if diet had changed dramatically, we are trying to identify factors motivating current high consumers of fruit and vegetables. Therefore a more 'health-conscious' group does not pose any real problems. Also, due to the large sample size, many differences between responders and non-responders are statistically significant despite the actual differences being very small.

The results of this study provide a unique insight into the food choice motivations and the behavioural change process, in relation to fruit and vegetable consumption, within this group of women.

\section{General food choice motivations}

The strongest determinants of general food choice in this sample of women were health and natural content. These two dimensions of the FCQ relate to the importance of food being 'nutritious' and 'good for you', as well as containing no additives or artificial ingredients. The sensory appeal and weight control dimensions were also strong motivators within this group. Few previous studies have tried to identify determinants of food choice solely in women. One panEU survey investigated both male and female attitudes to food, nutrition and health $(n=14331)$. The survey found that, both in the overall EU sample and in the UK sample, quality, price, taste, healthy eating and family constraints were the five most frequently mentioned influences on food choice ${ }^{6}$. If we compare these results we can see some quite different characteristics: in the current study, health issues, including natural content and weight control, are the top motivating factors, with sensory appeal also being considered important. However, in the EU sample sensory appeal is top of the list, including quality and taste, and price is a more highly influencing factor on food choice than is health.

Another study, utilising the FCQ to try to explain variation in dietary intake between meat eaters, low red meat eaters and vegetarians, found that their sample's strongest determinants of food choice were sensory appeal, price, health and convenience?. This group seems to have similar influences on food choice to the EU sample, both mentioning sensory appeal first, then price, followed by health. In the current study price was not seen as a prime motivating factor on food choice behaviour. This probably reflects the relatively high socio-economic group of women within this study. 


\section{Food choice motivations for bigh fruit and vegetable consumers}

Women appearing in the highest tertile of fruit and vegetable intakes were found to score higher on the health, natural content and ethical concern dimensions of the FCQ $(P<0.01)$. This is perhaps not suprising, as people who score high on the health and natural content dimensions will generally be more health-conscious and therefore are more likely to be aware of the health benefits of consuming adequate amounts of fruit and vegetables. Also the women who score high on all three of these dimensions - health, natural content and, especially, ethical concern - are more likely to be vegetarians. The study, referred to previously ${ }^{9}$, that attempted to explain variations in food choice between meat eaters, low red meat eaters and vegetarians found that the group who ate only small amounts of red meat scored highly on health, natural content, weight control and ethical concern factors of the FCQ. Whereas vegetarians only differed significantly on the ethical concern factors compared with the standard meat-eating group. Both of these groups, the 'healthconscious' and the vegetarians, have been found to have high intakes of fruit and vegetables in previous studies $^{12,13}$.

What was particularly interesting in this study was that higher consumers of fruit and vegetables had lower familiarity scores on the FCQ than lower consumers $(P<$ 0.01). The familiarity dimension of the FCQ seeks to find out if it is important to people that the food they eat 'is familiar' or 'is what I usually eat' or even 'is like the food I ate when I was a child'. It seems that the higher consumers of fruit and vegetables were less food-neophobic than the lower consumers; that is, that they are more likely to try new or novel foods, and they probably eat a wider variety of different foods ${ }^{14}$. This equates with previous data looking at lifestyle factors affecting fruit and vegetable consumption within the UKWCS. It was found that the higher consumers of fruit and vegetables consumed significantly more different varieties of fruit and vegetables, more than once per week, than did the lower consumers $^{12}$. It would make sense then that these people are perhaps less food-neophobic and are happy to experiment with new varieties, or cooking/preparation methods, of fruit and vegetables. It may therefore be important to make new varieties more available and certainly encourage new and different varieties of fruit and vegetables within the diet, in order to have any optimism over increasing intakes.

The high consumers of fruit and vegetables considered convenience concerns less of an issue than the low consumers, although this was not as statistically significant as the other dimensions mentioned $(P<0.05)$. Convenience concerns involved time available for preparation of food and shopping, availability of shops and simplicity of preparation and cooking. Convenience in terms of time constraints is a complex issue. It involves time available to go shopping and thus the perishable nature of foodstuffs, very applicable to fruit and vegetables. It also includes the time available for preparation and consumption of foods and therefore the convenience of the foodstuff itself. Previous studies show conflicting opinions on all aspects of time constraints and how they affect food choice decisions. An American investigation using focus group interviews found that fruit was viewed as convenient but was considered perishable and expensive when out of season. For vegetables, preparation time was considered to be a barrier to increased consumption ${ }^{15}$. However, among EU subjects, 'lengthy preparation' and 'healthy foods being more perishable' were considered as unimportant barriers to healthy eating. The main perceived barriers were still related to time though, including 'irregular working hours' and 'busy lifestyle'16. This conflicting evidence is probably due to the fact that time constraints will be more important to some groups of the population than others. In the present study, the high fruit and vegetable consumers rate issues around foods being able to be cooked very simply or easy to prepare as relatively unimportant. Perhaps the low consumers need to be educated in more quick and easy ways to incorporate fruit and vegetables into the diet.

The linear regression model was used to identify the strongest predictors of high fruit and vegetable consumption. After adjusting for age and education level, health and natural content were still the strongest predictors of high fruit and vegetable consumption $(P<0.01)$.

\section{Stages of change}

A clear relationship was observed between stages of change and actual consumption of fruit and vegetables, with women who were categorised as being precontemplators consuming the least amount of fruits and vegetables (3.74 portions per day) and those in the maintenance phase consuming a median of 7.11 portions daily $(P<0.01)$. All of the women categorised as being in one of the non-action phases - that is, pre-contemplation, contemplation and preparation - consumed less than five portions per day. On the other hand, the women in the action and maintenance groups consumed well over the recommended five portions per day: 6.29 portions and 7.11 portions, respectively. Previous studies have also reported a strong relationship between SOC for fruit and vegetable consumption and actual intakes ${ }^{17,18}$. These studies suggest that dietary interventions could benefit from a stage-tailored approach, so that people who are pre-contemplators need a different approach than do people who may be in the action phase. This implication is further backed up by the results of the present study, particularly when the FCQ scores within each SOC are considered. When the median FCQ scores were looked at within each stage some obvious relationships could be observed. Women in the maintenance and action phases had significantly higher median health, natural content, 
weight loss and ethical concern scores than in the other stages $(P<0.01)$. Conversely, women in the precontemplation phase tended to have the highest median convenience scores and women in the contemplation and preparation phases scored slightly lower on convenience than the pre-contemplators, but they were still higher than those who were in action or maintenance $(P<0.01)$. This information seems to suggest that if interventions were stage-tailored then those in the non-action phases would benefit from increased information on the health benefits of fruit and vegetables, including natural content and weight control issues. However, Diclemente suggests those individuals in pre-contemplation about a problem behaviour, in this case not consuming five portions of fruit and vegetables per day, are not even thinking about changing that behaviour and in fact may not even see the behaviour as a problem ${ }^{19}$. Therefore to give advice about exactly how to increase fruit and vegetables in the diet at this stage is going to be inappropriate and is likely to be seen as not relevant to the pre-contemplator. Instead it might be more appropriate to raise the awareness of the problem itself in order to move these people from the precontemplation phase into the contemplation and action phases. Once into the contemplation and action phases of the SOC model, interventions should also include additional information, as previously suggested in this paper, on more quick and easy ways to incorporate fruit and vegetables into the diet. If interventions are to be targeted towards people in the action phase, then more general information around convenience issues should be offered. Practical tips on making it easier to increase fruit and vegetable intakes on a daily basis, perhaps in the form of focus groups in order to offer support, would be preferential for achievement of the maintenance position.

\section{Limitations}

Reported fruit and vegetable intakes within the study group were higher than would normally be expected in the UK population. The median intake was 6.7 portions per person per day compared with the UK national average of $310 \mathrm{~g}$ per person per day, the equivalent of about 4 portions ${ }^{20}$. This may be partly due to the inherent problems of any self-reported data and, perhaps, because the sample was chosen to specifically include large numbers of vegetarians and vegans who are likely to consume greater quantities of fruit and vegetables. This has been shown to be true in the investigation into lifestyle factors affecting consumption in the UKWCS ${ }^{12}$.

It is also possible that overreporting contributed to the high fruit and vegetable intakes recorded. If this is true it may, at least partly, explain some of the relationships with the FCQ measures. For example, women appearing in the highest tertile of fruit and vegetable intakes were found to score higher on the health and natural content dimensions of the FCQ $(P<0.01)$. Perhaps these women tend to overreport fruit and vegetable intakes the most because they regard high intakes as desirable and are more aware of the health benefits of increased fruit and vegetable intakes.

It should be recognised that this particular cohort of women are generally well motivated, well educated and of a relatively high socio-economic status, and therefore some of the data may not be applicable to other subgroups of the population. Nevertheless the data give us a useful insight into the food choice motivations of a group of 'health-conscious' women with a high fruit and vegetable intake, and lead us to be able to make some suggestions as to what health promotion strategies should be based on.

\section{Conclusion}

In this particular group of women aged between 35 and 69 years, the most important motivating factors for food choice, within the high fruit and vegetable consumers, were health and natural content of the food. The findings of the study may help to create effective practical strategies for increasing overall consumption of fruit and vegetables among UK consumers. Any approach should be appropriately targeted towards the intervention population and this paper certainly suggests that it would be appropriate to use the SOC model in order to do this. Useful strategies would include raising the awareness of the problem in pre-contemplators, encouraging people at all other stages to experiment more with new varieties of fruit and vegetables, and trying to convince people that fruit and vegetables can be easy to prepare and fun and interesting to cook.

\section{References}

1 World Health Organization (WHO). Diet, Nutrition and the Prevention of Chronic Diseases. WHO Technical Report Series, No. 797. Geneva: WHO, 1990.

2 Department of Health. Saving Lives: Our Healthier Nation. London: HMSO, 1999.

3 Thompson RL, Margetts BM, Speller VM, McVey D. The Health Education Authority's health and lifestyle survey 1993: who are the low fruit and vegetable consumers? J. Epidemiol. Community Health 1999; 53(5): 294-9.

4 Lefebvre RC, Lurie D, Goodman LS, Weinberg L, Loughrey K. Social marketing and nutrition education: inappropriate or misunderstood? J. Nutr. Educ. 1995; 27: 146-50.

5 Cox DN, Anderson AS, Lean MEJ, Mela DJ. UK consumer attitudes, beliefs and barriers to increasing fruit and vegetable consumption. Public Health Nutr. 1998; 1(1): 61-8.

6 Institute of European Food Studies. A pan-EU Survey of Consumer Attitudes to Food, Nutrition and Health. Ireland: Institute of European Food Studies, 1996.

7 Steptoe A, Pollard TM, Wardle J. Development of a measure of the motives underlying the selection of food: the Food Choice Questionnaire. Appetite 1995; 25: 267-84.

8 Prochaska JO, DiClemente CC. Stages of change in the modification of problem behaviors. Prog. Behav. Modification 1992; 28: 183-218.

9 Pollard TM, Steptoe A, Wardle J. Motives underlying healthy 
eating: using the Food Choice Questionnaire to explain variation in dietary intake. J. Biosoc. Sci. 1998; 30: 165-79.

10 Crawley H. Food Portion Sizes. London: HMSO, 1994.

11 SPSS, Inc. SPSS for Windows: Release 9.O. Chicago, IL: SPSS, Inc., 1998.

12 Pollard J, Greenwood D, Kirk S, Cade J. Lifestyle factors affecting fruit and vegetable consumption in the UK Women's Cohort Study. Appetite 2001; 37: 71-9.

13 Kirk SF, Cade JE, Barrett JH, Conner M. Diet and lifestyle characteristics associated with dietary supplement use in women. Public Health Nutr. 1999; 2(1): 69-73.

14 Koivisto Hursti U, Sjoden P. Food and general neophobia and their relationship with self-reported food choice: familial resemblance in Swedish families with children of ages 7-17 years. Appetite 1997; 29: 89-103.

15 Heimendinger J, Van Duyn MA. Dietary behavior change: the challenge of recasting the role of fruit and vegetables in the American diet. Am. J. Clin. Nutr. 1995; 61(Suppl. 6): 1397S-401S.

16 Kearney KM, McElhone S. Perceived barriers in trying to eat healthier - results of a pan-EU consumer attitudinal survey. Br. J. Nutr. 1999; 81(2): 5133-7.

17 Laforge RG, Greene GW, Prochaska JO. Psychosocial factors influencing low fruit and vegetable consumption. J. Behav. Med. 1994; 17(4): 361-74.

18 Brug J, Glanz K, Kok G. The relationship between selfefficacy, attitudes, intake compared to others, consumption and stages of change related to fruit and vegetables. Am. J. Health Prom. 1997; 12(1): 25-30.

19 DiClemente CC. Motivational interviewing and the stages of change. In: Miller WR, Rollnick S, eds. Motivational Interviewing. Preparing People to Change Addictive Behavior. New York: The Guilford Press, 1991; 191-202.

20 Ministry of Agriculture, Fisheries and Food. National Food Survey 1998. London: The Stationery Office, 1999. 